\title{
A Saúde Coletiva no curso de Medicina da Universidade Estadual de Londrina: reflexões iniciais
}

\section{Community Health in the graduate medical course of the State University of Londrina: initial reflections}

João José Batista de Campos ${ }^{1}$ Paulo Eduardo Mangeon Elias ${ }^{2}$

\section{R E S U M O}

Este artigo analisa o enfoque da Saúde Coletiva no desenvolvimento curricular na graduação em Medicina da Universidade Estadual de Londrina (UEL), focalizando as diferentes visões de gestores, professores e estudantes sobre o processo de ensino-aprendizagem, introduzindo a análise da progressão do conhecimento em Saúde Coletiva no curso médico. Trata-se de um estudo qualitativo sobre a percepção dos entrevistados, tendo como população de estudo quatro gestores, quatro professores e um grupo focal de estudantes. Os resultados evidenciaram uma importante vinculação das atividades docentes da área junto à rede de serviços de saúde. No entanto, constata-se que os temas da área têm pequena inserção no currículo atual (5\%), não considerando os estágios do internato e a estratégia de ensino que vem sendo utilizada. Concluiu-se que os docentes do Departamento de Saúde Coletiva sempre tiveram uma participação de vanguarda nos processos de mudança no curso de Medicina da UEL, havendo um grande esforço em tomar a realidade dos serviços de saúde de Londrina como eixo articulador do ensino em Saúde Coletiva, na tentativa de contribuir para a formação de médicos com maior conhecimento da realidade e comprometidos com o SUS.

\begin{abstract}
A B S T R A C T
This qualitative study aims analyzing to which extent collective health is approached in the curriculum of the graduate course in medicine of the UE with special attention to the viewpoint of managers, professors and students regarding the teaching-learning process. To this purpose, four managers, four professors and a focal group of students were interviewed. The results revealed an important commitment of the teaching body in this area to the net of health services. However, internship and the teaching strategies in use apart, the subjects making part of this field participate with only $5 \%$ in the current curriculum. It was concluded that the professors of the Community Health Department have always held a vanguard position with regard to promoting changes in the medical course of the UEL and that there are strong efforts towards using the reality of the health services in Londrina as an articulating axle in the teaching of collective health so as to educate practitioners with greater awareness of the reality and commitment to the Unified Health System.
\end{abstract}

Recebido em: 10/07/2007 Reencaminhado em: 20/11/2007 Aprovado em: 25/11/2007

\footnotetext{
1 Universidade Estadual de Londrina, Paraná, Brasil.

2 Universidade de São Paulo, São Paulo, Brasil.
} 


\section{INTRODUÇÃO}

A primeira grande pergunta que se coloca para o professor de medicina quando se faz referência ao ensino e à aprendizagem da Saúde Coletiva é: qual a natureza desse objeto a ser ensinado ou aprendido?

A Saúde Coletiva tem sido definida como um campo de saber e de práticas que pressupõe a compreensão da saúde como um fenômeno eminentemente social, coletivo, determinado historicamente pelas condições e modos de vida dos distintos grupos da população. O debate teórico e epistemológico que existe hoje no campo da Saúde Coletiva contempla uma conceituação mais avançada da saúde, enquanto objeto de conhecimento e de intervenção, entendida como parte do "complexo saúde-doença-cuidado", que incorpora a historicidade das relações que a determinam, inclusive a relação dos indivíduos, grupos sociais e populações com o sistema de serviços de saúde ${ }^{1}$.

Ancorada nesta reflexão abrangente sobre a saúde, a Saúde Coletiva vem se configurando, especialmente no Brasil, como um campo de produção de conhecimentos e tecnologias, e como um "âmbito de práticas sociais"2.

Nesse sentido, a Saúde Coletiva se articula com um conjunto heterogêneo de movimentos ideológicos de reforma do ensino e da organização das práticas de saúde, redefinindo conceitual e politicamente algumas propostas e incorporando outras - a exemplo do que ocorreu com a Medicina Preventiva e com a Medicina Comunitária, nos primórdios da reflexão crítica na área, nos idos de 1970-80 e, mais recentemente, o diálogo com a Saúde da Família e o debate sobre as propostas e políticas de promoção da saúde ${ }^{1}$.

Na transição da década de 1980 para 1990 surgiu, numa reflexão de alcance internacional liderada pela OPS, uma abordagem do que se qualificou como "crise da saúde pública", definida como "a incapacidade da maioria das sociedades de promover e proteger sua saúde, na medida em que suas circunstâncias o requeiram"3. Na tentativa de abrir novas perspectivas de orientação para a saúde pública/coletiva, no movimento da promoção da saúde propôs-se a vertente conhecida como "cidades saudáveis". Ao mesmo tempo, a OMS promovia um debate sobre uma nova saúde pública, estimulando a reflexão no continente americano sobre "a teoria e prática da saúde pública"4,5.

Vale ressaltar os principais marcos da evolução desse campo de saber e práticas, inicialmente denominado Medicina Preventiva e Social, que se converteu num movimento até 1970. Ao se instaurar o movimento preventivista, estabeleceu-se um projeto pedagógico diferente do até então existente, que tivera seu principal modelo na formação de sanitaristas, via saúde pública. Re- gistre-se que a criação de um programa de residência médica na Faculdade de Medicina de Ribeirão Preto da USP, em 1962, pode ser citado como pioneiro na formação de um profissional com base na integração das ações preventiva e curativa ${ }^{6}$.

Até o final dos anos de 1960 e início de 1970, inclusive sob o impacto da Reforma Universitária (1968), apareceram outras iniciativas com o objetivo de reatualizar a formação de recursos humanos. Nessa década, alguns trabalhos estabeleceram uma contundente crítica às formulações e às práticas que antes haviam se voltado contra o modelo médico hegemônico; também se discutiram as diferenças entre Medicina Preventiva, Medicina Social e Saúde Pública ${ }^{7}$.

O trabalho de Arouca apresentou uma profunda análise crítica do projeto preventivista e assinalou: "A medicina preventiva realiza um trabalho de delimitação que, por um lado, afirma a sua identidade e a diferencia da própria medicina e, por outro lado, estabelece suas diferenças com a saúde pública e a medicina social". Nesta tese, o autor procurou, entre outros pontos, redefinir o campo de trabalho que, até então, tratava indistintamente o preventivo e o social ${ }^{8}$.

Em meados da década de 1970, as idéias sobre a Medicina Social foram analisadas em um documento da Opas que assume como entendimento ser um "campo de práticas e conhecimentos relacionados com a saúde, cuja preocupação principal é estudar a sociedade, analisar as formas correntes de interpretação dos problemas de saúde e da prática médica" ${ }^{\prime 9}$. Nesse sentido, o quadro conceitual que fornecia as bases para repensar esse campo de práticas era o de considerar "que, em cada formação social concreta, a educação cumpre um papel fundamental na reprodução da organização dos serviços de saúde e se cristaliza na re-atualização e preservação das práticas específicas, tanto nas dimensões do conhecimento quanto nas técnicas e no conteúdo ideológico" ${ }^{\prime \prime}$. Em outro ponto destacava: "é a própria estrutura da atenção médica que exerce um papel dominante através da estrutura do mercado de trabalho e das condições que circunscrevem a prática médica" ${ }^{\prime \prime}$.

Ao longo dos anos, a correta identificação da influência do mercado de trabalho sobre o processo de formação profissional degenerou, em alguns casos, em determinismo estrutural ${ }^{10}$. Essa forma mecânica de determinação da prática sobre o ensino merece ser questionada, como assinala Feuerwerker ${ }^{11}$, citando como exemplos a reforma do sistema de saúde inglês, feita imediatamente no pós-guerra ${ }^{12}$, e o modelo de formação médica adotado no Reino Unido, baseado no relatório Dawson e não na orientação flexneriana ${ }^{13}$. Ou seja, existiria a possibilidade de fazer prevalecer referencial e valores distintos dos hegemônicos, 
sem submeter a organização do sistema de saúde e da educação médica à lógica do capital - ainda que na vigência do capitalismo.

No campo da saúde, a consolidação do SUS no Brasil suscita novas e importantes questões diante da necessidade de oferecer atenção integral, resolutiva e de qualidade em todos os níveis do sistema. Por essa razão, o debate sobre formação e desenvolvimento dos profissionais de saúde vem ganhando importância crescente, já que o descompasso entre o perfil dos profissionais e as orientações do sistema de saúde é identificado como fator crítico para a consecução desses objetivos.

Apesar de ter havido, na última década, um descompasso entre os setores da saúde e da educação (o primeiro orientado por um movimento social democratizante, e o segundo orientado pelas diretrizes do estado mínimo), as novas diretrizes curriculares nacionais para os cursos universitários da área da saúde indicam caminhos para enfrentar esses desafios.

Essa confluência tornou-se possível por ser fruto de intensos debates e mobilização dos movimentos por mudança na educação dos profissionais de saúde, dos educadores, das associações educacionais, do Conselho de Secretários Municipais de Saúde e do Conselho Nacional de Saúde.

Um dos aspectos principais das diretrizes curriculares é a indicação de que na graduação deve haver maior vinculação da formação acadêmica às necessidades sociais de saúde, com ênfase no SUS. Indicam que os cursos de graduação devem ter como eixo do desenvolvimento curricular o processo saúde-doença em todas as suas dimensões e manifestações - considerando o cidadão, a família e a comunidade, integrados à realidade epidemiológica e social.

Entre as competências gerais dos profissionais, ressaltam as diretrizes que "os profissionais de saúde, dentro de seu âmbito profissional, devem estar aptos a desenvolver ações de prevenção, promoção, proteção e reabilitação da saúde, tanto em nível individual quanto coletivo"14.

De acordo com essas diretrizes, "a formação do profissional deve contemplar o sistema de saúde vigente no país, a atenção integral da saúde num sistema regionalizado e hierarquizado de referência e contra-referência e o trabalho em equipe"14.

Em conseqüência da necessidade de mudanças na formação profissional, na organização e nas práticas dos serviços, surgiram várias iniciativas orientadas à construção de agenda comum entre gestores municipais e instituições formadoras, visando a uma cooperação maior entre as universidades e os serviços de saúde, bem como à elaboração de projetos pedagógicos capazes de produzir perfis profissionais mais adequados ao sistema de saúde ${ }^{15}$. Metodologias ativas de ensino-aprendizagem, articulação entre trabalho e aprendizagem, prática ao longo de todo o curso e em todos os cenários em que ocorre o processo saúde-doença, compromisso com a resolução de problemas da realidade passaram a ser alguns dos elementos fundamentais das novas propostas para a formação profissional ${ }^{16}$.

Apesar do processo de construção do SUS ter alimentado diretamente o movimento por mudanças na educação dos profissionais de saúde, de modo geral, a participação da Saúde Coletiva na graduação médica não tem sido objeto de reflexão sistemática ${ }^{17}$.

O grande esforço de constituição do campo da Saúde Coletiva concentrou-se na pós-graduação e na produção de conhecimento. O lugar do ensino da saúde coletiva nos cursos de graduação da área de saúde tende a reproduzir os eixos disciplinares da pós-graduação: a Epidemiologia, o Planejamento e Gestão em Saúde, Ciências Sociais em Saúde e Saúde do Trabalhador. O que aparece quer como disciplinas específicas, quer como conteúdos embutidos em disciplinas mais gerais, ou como temáticas ou práticas em módulos interdisciplinares ${ }^{18}$.

Especificamente quanto ao ensino desses conteúdos, as novas diretrizes curriculares nacionais enfatizam a necessidade de incorporação do enfoque epidemiológico, populacional, na compreensão dos "determinantes sociais, culturais, comportamentais, psicológicos, ecológicos, éticos e legais" do processo saúde-doença, estabelecendo que a estrutura do curso de graduação tenha como um dos eixos do desenvolvimento curricular "as necessidades de saúde dos indivíduos e das populações", como consta da resolução aprovada pelo Conselho Nacional de Educação ${ }^{14}$.

Uma oficina de trabalho sobre Saúde Coletiva na graduação em Medicina, realizada pela Abrasco em dezembro de 2002 na Faculdade de Medicina da Santa Casa de São Paulo, revelou um importante desconhecimento dos professores de Saúde Coletiva em relação aos movimentos de mudança e às diretrizes curriculares. Também foi constatada uma significativa heterogeneidade em relação aos conteúdos de Saúde Coletiva atualmente presentes na graduação e um relativo afastamento do campo diante de questões e desafios concretos da área de educação e da própria construção do SUS ${ }^{19}$.

Entre as iniciativas promovidas no Brasil para a introdução de novas práticas de formação profissional em saúde, de profissionais tecnicamente preparados para prestar atenção integral à saúde e comprometidos com a solução dos problemas sanitários, destaca-se o Programa UNI, desenvolvido com o apoio da Fundação Kellogg em seis instituições de ensino superior brasileiras, em parceria com os sistemas locais de saúde e com organizações co- 
munitárias, para a implantação de mudanças na formação, na organização da atenção à saúde e na participação popular ${ }^{20}$.

Particularmente em relação às experiências apoiadas pelo Programa UNI, ressalta-se a do município de Londrina (PR), que vem sendo reconhecida no Brasil e no exterior como potencialmente promotora de mudanças significativas na formação de médicos. As novas propostas de orientação têm contemplado a interdisciplinaridade, a articulação entre ensino-serviços-comunidade, a ampla utilização de cenários de ensino extra-hospitalares e a alteração dos conteúdos, processos e relações.

No cenário das mudanças na graduação, essas experiências assumem um papel estratégico, justamente por trilharem os caminhos para consolidar os respectivos projetos de reforma na formação médica. Nesse sentido, a análise dos processos de implantação, a consideração das dificuldades enfrentadas, o estudo das alternativas propostas e, sobretudo, a avaliação dos primeiros resultados constituem focos de particular relevância na busca de alternativas para a (re)configuração até mesmo da própria crise da saúde pública ${ }^{21}$.

Estas reflexões iniciais sobre os 40 anos de ensino da Saúde Coletiva no curso de Medicina da UEL, particularmente dos últimos dez anos de currículo integrado, são de fundamental importância na identificação de estratégias que sirvam como subsídios para outras instituições que almejam fazer mudanças no campo da formação, validar alternativas de ensino para novos processos de formação e propor, com legitimidade, no âmbito nacional, princípios norteadores para as políticas de formação de médicos.

\section{OBJETIVO}

Este artigo analisa o enfoque da Saúde Coletiva no desenvolvimento curricular na graduação em Medicina da UEL por meio das diferentes visões de gestores, professores e estudantes sobre o processo de ensino-aprendizagem desenvolvido no curso e aprofundando a análise da progressão do conhecimento específico da área na graduação médica.

\section{METODOLOGIA}

O trabalho foi realizado por meio de um estudo de caso, com base em entrevistas realizadas com gestores, professores do Departamento de Saúde Coletiva e um grupo focal de estudantes do curso de Medicina, considerados estratégicos para o entendimento do processo de ensino e aprendizagem da Saúde Coletiva na graduação da UEL.

O conceito de "estudo de caso" adotado vem da tradição de pesquisa médica e psicológica, em que se recorre à análise minuci- osa de um caso individual que explica a dinâmica e a patologia de certa doença. Adaptado dessa tradição, o estudo de caso é uma das principais modalidades de análise das Ciências Sociais em Saúde ${ }^{22}$. O caso estudado é tipicamente o de uma organização, que, neste contexto, foi o curso de Medicina da UEL.

A população de estudo incluiu quatro gestores: o coordenador e o vice-coordenador do curso de Medicina, a chefe do Departamento de Saúde Coletiva e o diretor superintendente do Hospital Universitário. Também abrangeu quatro professores identificados como pessoas-chave, sendo estes alguns dos responsáveis pelas atividades mais diretamente relacionadas com a área de Saúde Coletiva e com intensa participação no curso. Finalmente, foi constituído um grupo focal com cinco estudantes de todas as séries do curso, exceto da primeira, também identificados como pessoas-chave, entre eles a atual presidente do Centro Acadêmico Samuel Pessoa (Casp).

Foi utilizado um questionário semi-estruturado para as entrevistas individuais com os gestores e professores selecionados, desenvolvido por meio de algumas questões norteadoras que tiveram três objetivos:

1. Conhecer o projeto pedagógico do curso de Medicina da UEL em seu processo de constituição, seus princípios fundamentais, suas diretrizes e seus mecanismos de sustentação.

2. Delinear o papel dos módulos mais diretamente relacionados com a Saúde Coletiva no conjunto do curso, buscando apreender seu significado no processo de formação dos médicos.

3. Caracterizar o perfil da área de conhecimento da Saúde Coletiva em suas dimensões estruturais, curriculares e pedagógicas, buscando apreender seus objetivos e sentidos.

Em relação aos estudantes, foi utilizada a técnica de grupo focal, que consistiu numa entrevista em grupo baseada em algumas perguntas norteadoras para a discussão, as quais envolveram a percepção dos estudantes nas várias atividades de ensino-aprendizagem desenvolvidas ao longo do curso sobre a área de conhecimento da Saúde Coletiva. Além disso, recuperaram-se todos os resultados dos testes de progresso dos estudantes de Medicina realizados desde o seu início no curso, referentes às questões específicas de Saúde Coletiva.

Portanto, a pesquisa foi feita com base na análise documental e nas entrevistas com os referidos personagens a partir de três eixos de investigação:

1. A Saúde Coletiva no contexto do curso médico.

2. A compreensão da Saúde Coletiva.

3. As dimensões de funcionamento da Saúde Coletiva. 
A análise desses dados se pautou pelo estabelecimento de categorias que emergiram da análise do discurso, isto é, pelo mapeamento e organização das unidades de significado. Com base no posicionamento dos sujeitos, foi possível estabelecer e confrontar, em cada eixo, diferentes dimensões da Saúde Coletiva: o currículo planejado, o vivido e o analisado. Mais do que a mera compilação ou descrição dos dados coletados, o que se pretendeu foi a reconstrução dos focos de sentido (pontos de consenso e tensão, modos de trabalho e dinâmicas de intervenção, expectativas e frentes de mudança) inerentes à rede discursiva, aspectos que, com certeza, podem traduzir a realidade em cada instituição.

\section{Bioética e coleta de dados}

A pesquisa foi aprovada pelo Comitê de Ética e Pesquisa da Universidade Estadual de Londrina através do Parecer $\mathrm{n}^{\mathrm{o}}$ 002/06, tendo-se esclarecido a todos os participantes os objetivos gerais do estudo e assegurado o sigilo das informações prestadas. Não houve qualquer conflito de interesse dos autores.

O grupo focal dos estudantes de Medicina foi realizado no Laboratório de Práticas Profissionais do Curso de Medicina da UEL, com a participação do moderador da discussão do grupo, além de duas observadoras.

A preparação e a execução das entrevistas individuais foram realizadas por um único entrevistador, gravadas e transcritas, o que viabilizou a análise dos dados utilizando-se a técnica de análise de conteúdo $^{23}$ por meio da triangulação metodológica ${ }^{24}$, na qual se empregam múltiplos meios de pesquisa para analisar o problema.

Esta metodologia é fundamental para verificar a propriedade das interpretações originadas a partir dos dados qualitativos coletados. A utilização desta estratégia na proposta de triangulação garante maior validade dos dados, pois os problemas e limitações de determinado método podem ser compensados pelo uso de outros métodos. Desta forma, as falas dos atores devem ser contextualizadas para que seja possível esclarecer e aprofundar alguns aspectos da realidade analisada ${ }^{25}$.

A análise documental do ensino da Saúde Coletiva na graduação em Medicina da UEL se concentrou nos principais textos legais que dizem respeito ao projeto pedagógico do curso. Trata-se de algumas resoluções das instâncias de deliberação mais importantes da Universidade, que refletem "os consensos construídos ao longo dos últimos anos em torno das inovações estruturais e metodológicas aprovadas em 1997 e implantadas no decorrer dos últimos 10 anos, de um curso que comemora em 2007, 40 anos de pleno funcionamento" 26 .

Além destes, analisaram-se os documentos produzidos pelos módulos relacionados com as temáticas da Saúde Coletiva, os relatórios de avaliação desses módulos e os resultados dos testes de progresso da Medicina referentes ao período de 1998 a 2006, que forneceram subsídios para a avaliação do desempenho dos estudantes na área de Saúde Coletiva especificamente. Todo esse material ajudou a compor a história do currículo atual do curso de Medicina da UEL.

\section{RESULTADOS}

Analisar as percepções dos professores, dos estudantes e dos gestores do curso pressupõe captar os diferentes olhares desses sujeitos sobre as várias características deste campo de saber e de práticas no curso de Medicina da instituição.

\section{O professor e o ensino em Saúde Coletiva na UEL}

Dos quatro professores entrevistados, um é homem e atua desde a criação da área de Saúde Coletiva há 37 anos em Londrina; os demais são mulheres que trabalham como docentes na instituição há mais de dez anos. Os quatro professores têm contrato em regime de tempo integral e dedicação exclusiva ao ensino, à pesquisa ou à extensão, sendo dois doutores e duas mestras em Saúde Coletiva.

Nos relatos, registrou-se que os professores de Saúde Coletiva iniciaram suas atividades acadêmicas em Londrina em 1969 e no ano seguinte se instalaram no Departamento de Medicina Preventiva, Higiene e Medicina do Trabalho da Faculdade de Medicina do Norte do Paraná. Após concurso para docente, foram contratados o professor Nelson Rodrigues dos Santos como chefe do Departamento e os professores Darli Antônio Soares e Maria Cristina Romariz Duarte.

A proposta original dos professores era desenvolver uma medicina integral, associando a medicina preventiva com a clínica por meio da organização da atenção primária à saúde. No segundo semestre de 1970, o trabalho prático se iniciou no primeiro Posto de Saúde Periférico, na Vila da Fraternidade, que passou a desenvolver não apenas ações de promoção de saúde e prevenção de doenças, como também atenção básica de saúde, sempre com a participação dos estudantes de Medicina desde a primeira turma do curso.

O objetivo dos professores, naquele momento inicial, era provocar alterações no ensino médico e na organização dos serviços de saúde nos níveis municipal e estadual, colaborando, assim, para mudanças em nível nacional. As estratégias utilizadas foram o desenvolvimento de trabalhos práticos ao lado de reflexões teóricas, procurando evitar o teoricismo, como também o desenvolvimento de atividades de forma integrada, para evitar o isolacionismo por meio de articulações internas e externas à Universidade. 
Nestes quase 38 anos de trabalho, os professores assumiram diferentes inserções institucionais. Em 1972, iniciou-se a área de Saúde Coletiva, como Departamento de Medicina Preventiva, que logo depois passou a ter outra denominação: Departamento de Medicina Geral e Saúde Comunitária. A partir de 1977, passou a se chamar Setor de Saúde Comunitária do Departamento
Materno-Infantil e Saúde Comunitária (Misc) e, finalmente em 2002, Departamento de Saúde Coletiva (Desc).

Na evolução histórica da área de Saúde Coletiva da UEL, é possível identificar algumas características marcantes e comuns ao longo de sua trajetória político-institucional:

QUADRO 1

Evolução histórica da área de Saúde Coletiva da UEL, 1969-2006.

\begin{tabular}{|c|c|}
\hline Ano & Evolução histórica \\
\hline 1969 a 1971 & Implantação de um campo de saber e prática inovadora em Londrina \\
\hline 1972 a 1976 & Consolidação e desenvolvimento institucional da Saúde Comunitária \\
\hline 1977 a 1981 & Desenvolvimento extra-institucional em nível local em Londrina \\
\hline 1982 a 1986 & Desenvolvimento extra-institucional em nível regional no estado do Paraná \\
\hline 1987 a 1990 & $\begin{array}{l}\text { Retomada do desenvolvimento institucional na UEL e criação do Núcleo de Estudos de Saúde Coletiva } \\
\text { (Nesco) e do Mestrado em Saúde Coletiva }\end{array}$ \\
\hline 1991 a 1993 & $\begin{array}{l}\text { Implantação do novo modelo de educação dos profissionais de saúde (UNI) no Centro de Ciências da } \\
\text { Saúde da UEL }\end{array}$ \\
\hline 1994 a 2000 & $\begin{array}{l}\text { Processo de consolidação do novo modelo de educação dos profissionais de saúde associado à } \\
\text { construção de um novo modelo de atenção à saúde }\end{array}$ \\
\hline 2001 a 2002 & Mudança institucional com a criação do Departamento de Saúde Coletiva \\
\hline 2003 a 2006 & Implantação efetiva do Departamento de Saúde Coletiva na UEL \\
\hline
\end{tabular}

De 1977 a 1986, os professores tiveram um papel fundamental no início do processo de implantação das Unidades Básicas de Saúde em Londrina e desempenharam importante função no desenvolvimento da proposta e das primeiras experiências, que foram se disseminando por toda a região. Este fato histórico deve ser destacado na trajetória dos professores, pois, de certa forma, mudou a política de saúde no estado do Paraná.

Os professores orientavam suas práticas acadêmicas e políticas por um referencial metodológico, nem sempre claramente explicitado. No entanto, o marco teórico adotado serviu, em larga medida, para auxiliar na sobrevivência institucional e no crescimento político do grupo, que era muito reduzido em seu início, com menos de dez docentes.

Como realidade concreta, as características do trabalho desenvolvido pelos docentes em Londrina não permitem um nítido enquadramento em qualquer das classificações propostas na área de Saúde Coletiva. Em outras palavras, não se enquadram formalmente no modelo mínimo legal, ou no modelo liberal ou no modelo racionalizador desenvolvido pelo Peses ${ }^{27}$. Igualmente, não se adaptam a propostas preventivistas, racionalizadoras ou teórico-críticas.

Os professores da Saúde Coletiva da UEL estabeleceram, ao longo do tempo, um consenso em torno da finalidade de contribuir para o processo de transformação das instituições de ensino e de atenção à saúde, visando à construção de um sistema social mais justo e inclusivo, por meio de mudanças nas formas de organização e nas práticas e resultados das mesmas.

Desde 1998, no novo currículo integrado de Medicina, os docentes do Departamento de Saúde Coletiva passaram a atuar mais intensamente em quatro módulos do curso, que, somados, representam $5 \%$ da carga horária total do curso:

- Abrangência das Ações deSaúde, módulo transversal de quatro semanas que encerra a primeira série dos estudantes do curso e tem por objetivos conhecer as relações entre a saúde e a organização social, as disposições legais que orientam o desenvolvimento do Sistema Único de Saúde (SUS), analisar as contribui- 
ções da Epidemiologia para a compreensão dos processos da saúde e da doença, além de reconhecer as modalidades e condicionantes que existem na organização da atenção à saúde ${ }^{26}$.

- Práticas de Interação Ensino, Serviço e Comunidade I, módulo longitudinal desenvolvido ao longo de toda a primeira série, às quartas-feiras à tarde, que pretende que os estudantes vivenciem diferentes práticas do cuidado em saúde aos pacientes cadastrados nas dez Unidades Básicas de Saúde selecionadas, com base na observação das condições de vida da população do território, no enfoque da Saúde da Família e com a construção de parcerias que respondam às necessidades de saúde dos usuários ${ }^{26}$.

- Doenças Resultantes da Agressão ao Meio Ambiente, módulo transversal de quatro semanas que inicia a segunda série do curso e tem por objetivo capacitar o estudante a reconhecer o impacto de alterações ecológicas, diretas ou indiretas, sobre a saúde humana, decorrentes da interação entre os grupos sociais e destes com a natureza ${ }^{26}$.

- Práticas de Interação Ensino, Serviço e Comunidade II, módulo longitudinal desenvolvido ao longo da segunda série do curso, que tem por objetivo conhecer o sistema local de saúde e as atividades em primeiro nível de atenção no município de Londrina, avaliando o desenvolvimento de uma das ações programáticas da Unidade Básica de Saúde ${ }^{26}$.

No internato médico, que se desenvolve nos últimos dois anos do curso, com 24 meses de duração a partir da quinta série, não existe uma atuação específica dos docentes da Saúde Coletiva como preceptores em nenhum dos 35 estágios supervisionados. No entanto, muitas outras práticas de Saúde Coletiva são desenvolvidas nesta importante fase de formação dos estudantes, como apontam as Diretrizes Curriculares Nacionais.

\section{O estudante e a aprendizagem em Saúde Coletiva na UEL}

Neste corpo de análise, destacaram-se algumas discussões que pareceram mais significativas no grupo focal dos estudantes.

Quando perguntada ao grupo sua opinião acerca da Saúde Coletiva no curso, uma estudante enfatizou a necessidade de abordar, nos módulos voltados para a prática, problemas mais concretos relacionados à profissão médica que pudessem estimular os estudantes a procurarem esta área em sua especialização pós-graduada.

Durante o debate sobre onde foram trabalhadas mais intensamente as práticas de Saúde Coletiva ao longo do curso, o grupo pareceu ter dificuldade em identificar os "conteúdos" da Saúde Coletiva nos módulos em que eles de fato acontecem. Citam o Centro Acadêmico, entre outros, como espaço de aprendizagem sobre o SUS.
Apresentam também uma visão de que o Pronto Socorro do Hospital Universitário lota porque a Saúde Coletiva não cumpriu o seu papel antes do internato médico, orientando a população sobre as diferenças entre a Atenção Básica, Secundária e o Hospital Terciário, que acaba virando um "postão".

O modelo curricular do curso de Medicina foi apontado por todos como sendo centrado no estudante. No entanto, foi consenso que os instrutores de prática dos módulos de interação Ensino, Serviço e Comunidade têm significativo papel no sucesso ou no fracasso da atuação dos grupos multiprofissionais nos territórios de suas respectivas Unidades Básicas de Saúde.

Nas discussões que acontecem nos grupos tutoriais ao longo do curso, muitos tutores abortam os conteúdos de Saúde Coletiva ou não problematizam adequadamente as discussões dos problemas de papel quando estes são trazidos da realidade, comentário de um dos internos presentes no grupo focal.

Outro aspecto apontado pelos estudantes é a desvalorização da Saúde Coletiva pelos veteranos do curso em relação aos calouros. Isso contribui com uma visão negativa da área, que, por outro lado, é muito valorizada nos congressos de educação médica e em outros eventos dos quais os estudantes participam durante a graduação e que interferem positivamente na construção de sua visão acerca da área, no final do curso.

Quando questionados sobre como obter maior inserção do estudante no processo de trabalho das equipes de Saúde da Família, todos se referiram ao módulo de Práticas de Interação, Ensino, Serviço e Comunidade 1 (PIN), desenvolvido em conjunto nas primeiras séries dos cursos de Medicina e Enfermagem da UEL. Todos argumentaram sobre a relevância dos problemas estudados, a acolhida das equipes das Unidades Básicas de Saúde e o papel atribuído aos estudantes, entre outros aspectos. Ao final, uma estudante lançou uma síntese acolhida pelo grupo: a Saúde Coletiva é o PIN.

Sobre o trabalho multiprofissional realizado especialmente na relação entre os estudantes de Medicina e Enfermagem, foi destacada a importância do instrutor na construção da ponte entre eles. É interessante observar que o grupo refere o papel ativo do estudante, viabilizado pelo currículo, mas contraditoriamente credita ao instrutor as parcerias construídas, a dinâmica do grupo, a explicação sobre a importância do SUS. Referem-se ao instrutor como o responsável pela efetivação das ações e não valorizam seu próprio papel na constituição da equipe acadêmica de saúde que, inclusive, irá atuar por dois anos consecutivos naquele mesmo bairro da cidade de Londrina.

Quanto à questão da avaliação, segundo os estudantes, está centrada no professor-instrutor e circula entre o cognitivo e o atitudinal na primeira série do curso, mas depois os estudantes só 
referiram o cognitivo. Destacou-se a falta de devolutiva dos instrutores, gerando um grande debate sobre o conceito de avaliação. Alguns defendem que ela é parte do processo de ensino-aprendizagem e deve ser contínua e permanente, incluindo as relações que se estabelecem com a equipe e os usuários como parte desse processo. Outros, ao afirmarem que a matéria dada deve ser avaliada no final do módulo, porque o estudante "só corre atrás quando é cobrado", demonstram certa confusão entre o que seja avaliação cognitiva e formativa.

Em síntese, não houve consenso em alguns assuntos, cada um deu sua opinião, e os resultados obtidos sugerem que as informações obtidas foram suficientes para a caracterização de um olhar importante do processo de formação do profissional médico, na perspectiva da aprendizagem da Saúde Coletiva.

\section{Avaliação dos Testes de Progresso da Saúde Coletiva}

O processo de avaliação do desempenho dos estudantes do curso de Medicina da UEL pode ser inicialmente estimulado por um projeto de pesquisa desenvolvido pela Comissão Interinstitucional Nacional de Avaliação do Ensino Médico (Cinaem), caracterizado como um estudo multicêntrico e chamado de Estudo de Coorte (avaliação dos formandos), realizado no início de 1996 por meio do Teste de Qualificação Cognitivo Inicial (TQCI) e, no final de 1997, pelo Teste de Qualificação Cognitivo Final (TQCF) que contou com a participação de 42 escolas médicas brasileiras.

A pesquisa desenvolvida pela Cinaem foi muito importante para Londrina, pois os estudantes que participaram da investigação integravam a última turma do curso tradicional de Medi- cina, anterior à reforma curricular introduzida em 1998. Os resultados de seu desempenho mostraram que a média de $48 \%$ de acertos foi discretamente superior à nacional (43\%) no teste inicial, mantendo-se superior à de seus colegas no final, com 55\%, sendo que a proporção média de acertos na área específica de Saúde Coletiva para o Brasil foi de $51 \%{ }^{28}$.

O estudo evidenciou, por um lado, que o desempenho cognitivo médio dos nossos estudantes em comparação com as outras escolas médicas brasileiras era bom. No entanto, constatou-se uma ausência de tradição e, conseqüentemente, de estrutura para a avaliação de conhecimentos aplicados, habilidades e atitudes, sendo que o curso da UELnão aplicou a Ficha Estruturada de Avaliação Prática proposta pela Cinaem² ${ }^{28}$.

Diante desta realidade, a Comissão de Avaliação, o Colegiado do Curso de Medicina e a Direção do Centro de Ciências da Saúde da UEL, liderados pelo professor Kazuhiro Ito, decidiram aplicar, em 1998, o primeiro Teste de Progresso da Medicina (TPMed $)^{29}$ a todos os estudantes da primeira à sexta série do curso. Essas questões do TPMed constituíram o Teste de Qualificação Cognitivo Inicial da Cinaem, aplicado em 1996 apenas aos internos da quinta série na UEL. Além disso, introduziram a avaliação de habilidades clínicas por exame objetivo estruturado em estações, com emprego de pacientes padronizado ${ }^{30}$, tendo sido realizadas, ao longo dos dez anos de currículo integrado, mais de 50 Osces (Objective Structured Clinical Examination) ${ }^{31}$ adaptadas ao nosso contexto.

Os resultados dos testes de progresso da Saúde Coletiva realizados de 1998 a 2006 estão apresentados na Tabela 1.

\section{TABELA 1}

Percentuais médios de testes respondidos corretamente em Saúde Coletiva por ocasião dos Testes de Progresso do Curso de Medicina da UEL de 1998 a 2006

\begin{tabular}{ccccccc}
\hline Ano & $1^{\text {a }}$ série & $2^{\text {a }}$ série & $3^{\text {a }}$ série & $4^{\text {a }}$ série & $5^{\text {a }}$ série & $6^{\text {a }}$ série \\
\hline 1998 & 42,9 & 37,6 & 49,1 & 51,5 & 41,9 & 48,0 \\
$1999 / 1$ & 13,6 & 23,4 & 25,6 & 40,6 & 36,1 & 32,3 \\
$1999 / 2$ & 25,4 & 40,0 & 41,7 & 41,7 & 42,2 & 58,8 \\
2002 & 39,2 & 48,4 & 53,1 & 53,1 & 51,9 & 63,8 \\
2003 & 52,9 & 61,6 & 60,7 & 64,9 & 65,3 & 74,0 \\
2004 & 48,1 & 66,6 & 64,5 & 64,8 & 69,0 & 72,6 \\
2005 & 41,6 & 49,1 & 46,6 & 47,0 & 51,0 & 59,1 \\
2006 & 46,2 & 46,9 & 48,1 & 49,1 & 54,4 & 60,3 \\
\hline
\end{tabular}


Em 1998, a média da quarta série não apresentou diferença estatisticamente significante em relação a terceira e a sexta séries, mas foi estatisticamente maior do que a quinta série, e a média da sexta série não diferiu da primeira série. Em 2002, a primeira e a sexta séries diferiram de todas as demais, mas a segunda, a terceira, a quarta e a quinta séries não foram diferentes entre si. $\mathrm{O}$ melhor desempenho dos estudantes foi obtido no teste realizado em 2003, fato que, aliado aos mais de 50\% de acerto dos calouros, sugere que as questões apresentaram baixo grau de dificulda$\mathrm{de}^{29}$, o que se repete de certa forma em 2004. Em 2005, verifica-se uma pequena queda na terceira série, e em 2006 mantém-se o padrão de crescimento no teste elaborado e realizado em conjunto com outras dez escolas médicas brasileiras articuladas entre si com o apoio da Abem.

\section{FIGURA 1}

Curvas de crescimento cognitivo dos Testes de Progresso do Curso de Medicina da UEL aplicados de 1998 a 2006.

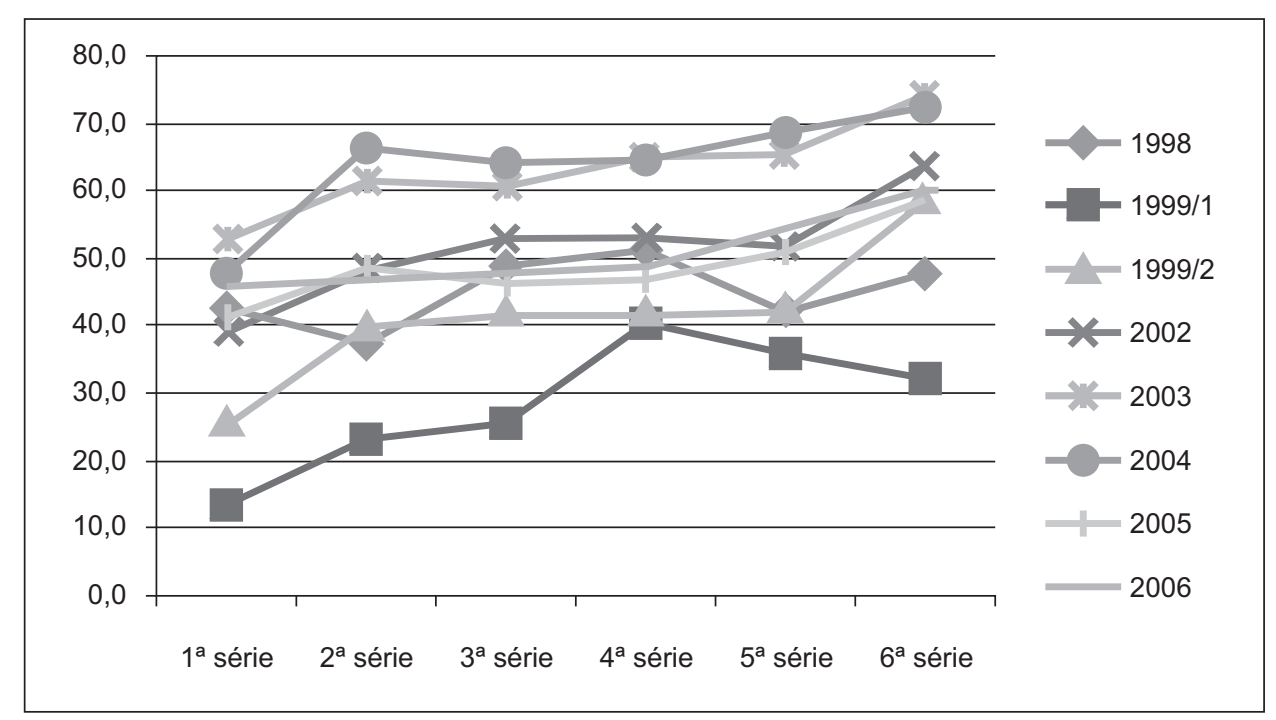

Como os distintos testes não foram elaborados seguindo um mesmo critério e com igual grau de dificuldade, eles medem conhecimentos diferentes, ou seja, não existe uma clara tendência em relação ao desempenho dos estudantes na área de Saúde Coletiva. Os diferentes níveis de adesão dos estudantes ao teste, as magnitudes dos crescimentos cognitivos e os tipos de crescimento das curvas dos oito testes realizados demonstram variações nem sempre muito fáceis de explicar. Após alguns anos de estudos sobre o teste de progresso em Londrina, aprendeu-se que a análise estatística que pode ser feita é apenas comparar o desempenho dos estudantes nas diferentes séries em um mesmo teste, mas não se pode fazer uma comparação entre os diferentes testes.

\section{O gestor e o ensino em Saúde Coletiva na UEL}

Os quatro gestores entrevistados ocupam seus cargos há mais de um ano, tendo trajetórias profissionais variadas: três possuem muita experiência administrativa e um vivencia sua primeira oportunidade de gestão. As atuais estruturas formais de gestão acadêmica e administrativa do curso de Medicina da UEL são constituídas por um Colegiado Pleno de 96 professores, que elegem uma Comissão Executiva de oito membros, sendo que os estudantes indicam três representantes, o que totaliza 11 pessoas.

No curso de Medicina da UEL, diferentemente de outros cursos de graduação, existem quatro comissões de apoio: Avaliação, Educação Permanente Docente, Apoio Psicopedagógico do Docente e Discente, e Acompanhamento Curricular. Cada uma delas é constituída por cinco docentes indicados pelos departamentos envolvidos com o curso e um estudante indicado pelo Centro Acadêmico Samuel Pessoa. Elas assessoram a Comissão Executiva e trabalham junto com seus membros.

A gestão do curso é assumida em grande parte pelo Colegiado de Medicina, que vem consolidando uma infra-estrutura de apoio à implementação das mudanças curriculares. Atualmente, os departamentos e a direção do Centro de Ciências da Saúde têm acompanhado o processo sem direcionalidade. O coordenador do curso tem assento no Conselho do Centro de Ciências da 
Saúde e na Câmara de Graduação, que é uma das Câmaras do Conselho de Ensino, Pesquisa e Extensão da Universidade.

Todos os entrevistados apontaram a atuação dos docentes de Saúde Coletiva em Londrina como muito intensa e responsável, porém se avançou pouco na discussão da integralidade do currículo como ferramenta para a operacionalização de inovações curriculares que pudessem ajudar a responder questões como: o que é de fato integração curricular para além da integração disciplinar nas metodologias ativas? Como integrar os saberes dos diferentes conteúdos com suas respectivas habilidades na perspectiva da integralidade no atendimento ao paciente?

\section{CONSIDERAÇÕES FINAIS}

Os docentes do Departamento de Saúde Coletiva sempre tiveram uma participação de vanguarda nos processos de mudança no curso de Medicina da UEL. Associado a uma busca de estreita articulação com os docentes das áreas clínicas e cirúrgicas, isto contribuiu muito para que pudessem desempenhar um papel de liderança ao longo dos 40 anos do curso de Medicina, em especial nos últimos dez anos do currículo integrado.

Existe em Londrina uma concepção própria de Saúde Coletiva que em muito determinou essa situação, além de muita habilidade política desses docentes, que nunca se isolaram, apesar de todas as diferenças de concepção de Medicina, de políticas de saúde ou de divergências ideológicas sobre a política nacional. Pelo contrário, eles tiveram sempre uma atitude gregária, preocupados em tomar parte das lutas comuns a todos, participando da criação do Sindicato dos Professores e se envolvendo com os clínicos e cirurgiões nas questões referentes ao Hospital Universitário, cujo diretor superintendente, reeleito, é, inclusive, um dos docentes do Departamento de Saúde Coletiva.

Os estudantes reconhecem a presença e o desenvolvimento pouco convencional da área de Saúde Coletiva, uma vez que não existem disciplinas no currículo do curso e, sim, módulos interdisciplinares, trabalho multiprofissional com práticas nas Unidades Básicas de Saúde desde o primeiro ano, o que tem possibilitado uma participação mais ativa dos alunos e a realização de mudanças mais consistentes, com maior sustentação ao longo do tempo, apesar das dificuldades e instabilidades políticas que afligiram o curso nesta trajetória e da dependência do professor referida no grupo focal.

Uma das principais características deste currículo se traduz no grande esforço em tomar a realidade dos serviços de saúde de Londrina como eixo articulador do ensino em Saúde Coletiva para formar, assim, médicos conhecedores da realidade e potencialmente mais comprometidos com a solução dos problemas da população.

\section{REFERÊNCIAS}

1. Teixeira CF. Ensino da Saúde Coletiva na graduação. Boletim da Abem, v. 31, n.3, maio/jun. 2003.

2. Paim JS, Almeida Filho N. A crise da Saúde Pública e a utopia da Saúde Coletiva. Salvador: Casa da Qualidade; 2000.

3. Organización Panamericana de la Salud. Sobre la teoria y prática de la salud pública: um debate, multiples perspectivas. Washington; 1993.

4. Organización Panamericana de la Salud. La crisis de la salud pública: reflection para el debate. Washington; 1992. (Publicación científica n⿳0 540).

5. Ferreira JR, Buss PM. O que o desenvolvimento local tem a ver com a promoção da saúde? In: Zancan L, Bodstein R, Marcondes WB orgs. Promoção da saúde como caminho para o desenvolvimento local: a experiência em Manguinhos - RJ. Rio de Janeiro: Abrasco/Fiocruz; 2002.

6. Nunes, ED. Saúde Coletiva: história de uma idéia e de um conceito. Saúde e Sociedade 1994; 3(2): 5-21.

7. Garcia JC. La educación médica en América Latina. Washington: Organización Panamericana de la Salud; 1972.

8. Arouca ASS. O dilema preventivista: contribuição para a compreensão e crítica da medicina preventiva. São Paulo; 1975 Tese [Doutorado] - Universidade Estadual de Campinas.

9. Organización Panamericana de la Salud. Enseñanza de la medicina preventiva y social: 20 años de experiencia latinoamericana.Washington;1976. (Publicación científica no 234).

10. Almeida MJ. Educação médica e saúde: possibilidades de mudança. Londrina: Eduel; Rio de Janeiro: Abem; 1999.

11. Feuerwerker LCM. Além do discurso de mudança na educação médica: processos e resultados. São Paulo: Hucitec; 2002.

12. Testa M. Pensamento estratégico e lógica de programação: o caso da saúde. São Paulo: Hucitec/Abrasco; 1995.

13. Flexner A. Medical Education in the United States and Canada. New York: The Carnegie Foundation for the Advancement of Teaching; 1910.

14. Almeida MJ, Maranhão E. Diretrizes curriculares nacionais para os cursos universitários da área da saúde. Londrina: Rede Unida; 2003.

15. Rede Unida, Conasems. Construindo a agenda dos gestores municipais: cooperação entre as universidades e serviços de saúde. Belo Horizonte; 2003. (Oficina de trabalho).

16. Almeida MJ, Coord., Pereira LA, Turini B, Nicoletto SCS, Campos JJB, Rezende LR, Mello PL. Formação dos profissi- 
onais de saúde no Paraná e a implantação das diretrizes curriculares nacionais. [Paraná]; 2005. (Relatório final do Projeto de Pesquisa financiado pelo CNPq no 47.4029/2003-4).

17. Forster AC, Passos ADC, Dall-Fabbro AL, Laprega MR. Transformation and trends in preventive and social medicine education at the undergraduate level in a Brazilian medical school. Gac Sanit. 2001; 15(6): 519-22.

18. Cyrino EG. Contribuições ao desenvolvimento curricular da Faculdade de Medicina de Botucatu: descrição e análise dos casos dos cursos de Pediatria e Saúde Coletiva como iniciativas de mudança pedagógica no terceiro ano médico. São Paulo; 2002. Tese [Doutorado] - Universidade Estadual Paulista.

19. Marsiglia RG, Feuerwerker LCM, Carneiro JrN. Oficina Nacional de Trabalho. O Ensino de Saúde Coletiva na Graduação Médica, São Paulo: Faculdade de Ciências Médicas da Santa Casa; 2002. (Relatório final).

20. Kisil M, Chaves M. Programa UNI: uma nova iniciativa na educação dos profissionais de saúde. Battle Creek: Fundação W. K. Kellogg; 1994.

21. Campos JJB. Como abrir caminhos para a transformação do ensino médico no Brasil. Rev. Bras. Educ. Med. 1999; 23(2/3): 11-20.

22. Becker HS. Métodos de pesquisa em ciências sociais. São Paulo: Hucitec; 1997.

23. Bardin L. Análise de conteúdo. Lisboa: Edições 70; 1977.

24. Minayo MCS. O desafio do conhecimento. São Paulo: Hucitec; 1992.

25. Pontes RHP. Reforma curricular no ensino médico: estudo de caso de uma escola de medicina. São Paulo; 2001. Tese [Doutorado] - Universidade de São Paulo.

26. Universidade Estadual de Londrina. Centro de Ciências da Saúde. Colegiado do Curso de Medicina. Atos legais de consolidação do currículo integrado / Colegiado do Curso de Medicina, Centro de Ciências da Saúde, Universidade Estadual de Londrina. Londrina: Universidade Estadual de Londrina; 2006.

27. Peses. Programa de Estudos Sócio-Econômicos em Saúde (Peses): Investigação Nacional sobre o Ensino da Medicina Preventiva. Rio de Janeiro; 1978. (Relatório de Pesquisa).

28. Piccini RX, Facchini LA, Santos RC. Preparando a transformação da educação médica brasileira. Projeto Cinaem III Fase; 2000. (Relatório 1999-2000).

29. Universidade Estadual de Londrina. Centro de Ciências da Saúde. Colegiado do Curso de Medicina. Resultados dos Testes de Progresso da Medicina realizados no período de 1998 a 2002; 2003.

30. Troncon LEA et al. Implantação de um programa de avaliação terminal do desempenho dos graduandos para estimar a eficácia do currículo na Faculdade de Medicina de Ribeirão Preto. Rev. Assoc Med Bras. 1999; 45(3): 217-24.

31. Harden RM. Assessment of clinical competence using objective structured clinical examination. Br Med J. 1975; 1(5955): 447-51.

\section{CONFLITOS DE INTERESSE}

Declarou não haver.

\section{ENDEREÇO PARA CORRESPONDÊNCIA}

João José Batista de Campos

Núcleo de Estudos de Saúde Coletiva da UEL

Rua Robert Koch, 60 - Vila Operária

86038-350 - Londrina - Paraná

E-mail: jocampos@uel.br 